\title{
The Study and Analysis of the Sense of Acquisition of the Ethnic College Students
}

\author{
Wang Yadong \\ Nanchang Institute of Science \&Technology, Nanchang, Jiangxi, China \\ Email:1773381558@qq.com
}

Keywords: ethnic college students; sense of acquisition; exploration and analysis

\begin{abstract}
Sense of acquisition is the hot words of the contemporary society. The ethnic college students studying in Jiangxi Province are the beneficiaries of the favorable ethic policies of our country. Therefore, their sense of acquisition are the strongest. The essay focuses on their senses of acquisition in all the aspects of their life, based on the theories of cognitive psychology, cognitive neurology, data processing, pedagogy, etc. It employs empirical and factors analysis methods and obtains 57 factors through an internet questionnaire survey tool. Then it analyzes these factors by employing factor exploration and factor fitting methods, and it has finally proved six key factors of their senses of acquisition.
\end{abstract}

\section{Literature Review on the Sense of Acquisition}

It is only four years since the term "sense of acquisition" was coined in 2013. However, the concept of "sense of acquisition" has now been deeply rooted in people's hearts and permeated every field of society and every aspect of people's life. "Sense of acquisition" is a product of reform and opening up, and with a distinct brand of the time. Theoretical research on the sense of acquisition started in 2015.Based on the search term "sense of acquisition", the author searched the CNKI and found 1,229 essays of various kinds from 2015 to July 2017.Among them, 164 essays are from Chinese core journal criterion of PKU and 135 ones are from the sources of CSSCI. In terms of number, the annual growth momentum is very rapid: 186 in 2015, 616 in 2016 and 427 in 2017.In terms of quality, the number of high-quality essays has increased significantly: 19 core journal criterion of PKU in 2015, 73 in 2016 and 72 in 2017; 11 from CSSCI sources in 2015, 57 in 2016 and 67 in 2017.

Regarding the theme and research content of the essays, their characteristics are quite obvious. That is, it tightly revolves around a center and a theme. A center: To improve people's sense of acquisition by what they should do, and how they should do. One theme: To reform and open up. That is, focusing on issues closely related to reform and opening up, deepening reform, combating corruption and upholding integrity, governing the party and governing the country, and improving people's livelihood. Specifically, the essays can be divided into the following categories: Anti-corruption group: This kind of essays mainly describes how to improve people's sense of acquisition through political reforms, such as ruling the party and governing the country, anti-corruption and building a clean government. Deepening reform group: These essays explore how institutions in various fields can deepen reforms to improve sense of acquisition. Theory exploration group: These essays explore from deep level the concept, definition, implication, connotation, meaning, value and other aspects of sense of acquisition, and reveal its essence. Education group: These essays explore how education departments reform their institutions, policies, ideas and methods to enhance the sense of acquisition of the teachers and students. People's livelihood group: These essays pay close attention to the reforms of all kinds of social institutions that are closely related to people's livelihood, improve efficiency and improve people's livelihood in order to improve people's sense of acquisition. Agriculture, rural areas and farmers group: These essays pay attention to agricultural and rural reform to improve farmers' sense of acquisition. 
Regarding research trend, there are mainly the following aspects: The 2015 essay focuses on specific measures to improve people's sense of acquisition .From 2016 to 2017, there are many essays discussing the connotation and essence of sense of acquisition. Researching areas have been gradually expanded from a limited areas to wider fields. The concerning targets have shifted from urban residents to school students, from hospital patients to rural farmers.

Shortcomings in the present study: The research methods are relatively simple and especially lacks empirical ones. Most essays are from theory to theory, while few essays are from data to conclusion. Researching methods need improving. Sense of acquisition is closely related to the brain and nerves. So it has something to do with cognitive science such as cognitive psychology, cognitive linguistics and cognitive neurology. Limited by the conditions and apparatus, the essays that use high-tech instruments to study the sense of acquisition are basically blank. Researching trend prediction: The researching field will continue to expand. With the deepening of reform and opening up, the research of sense of acquisition will keep developing and deepening in a synchronous manner as it expands to unknown areas. Researching perspectives will become diversified. With the deepening of the research on the theories of sense of acquisition, the theories will be considerably enriched and the research perspective will become more and more abundant and novel.

\section{The Basic Features and Models of the Sense of Acquisition}

\subsection{The basic features of the sense of acquisition}

The marginal effect of sense of acquisition: It refers to the fact that if a certain kind of gain is continuously increased, the output of the sense of gain will be gradually reduced. The critical point effect of sense of gain: It refers to the fact that the sense of acquisition increases with the increase of acquisition at the beginning, and then reaches a relative peak, that is, the critical point of sense of acquisition. After that, if the gain go on increasing, the sense of gain will keep decreasing. The relativity of sense of gain: All the sense of gain is relative and not absolute. It is relative in comparison with others. The dynamic nature of sense of acquisition: The sense of acquisition is not fixed, it is unstable and will change with time.

\subsection{The basic models of the sense of acquisition}

It is assumed that the concrete or abstract acquisition can be realized through the perception of human sensory organs, and initial acquisition can be generated. This acquisition then goes through the brain for information processing and psychology transformation, and eventually produces a sense of acquisition. Therefore, the basic model of sense of acquisition can be summarized as the following: concrete or abstract acquisition $\rightarrow$ sensor $\rightarrow$ acquisition $\rightarrow$ information processing $\rightarrow$ psychological transformation $\rightarrow$ sense of acquisition.

\section{The Research Hypothesis and Proof of the Sense of Acquisition}

Hypothesis 1: The sense of acquisition is proportional to the actual gain before reaching the critical point, that is, the sense of acquisition is equal to the actual accumulated gain. This hypothesis can be expressed by a formula of positive proportionality function, and the graph is "/". Hypothesis proof through psychological experiment 1: Sense of acquisition of learning English words. Experimental design: This experiment adopts observation method, control method and cognitive psychology oral report method. The experimented students were divided into two groups. The first group was taught 10 new words, and the second group was taught 20 new words. Both groups were taught with the same teaching method.

The test results showed that the listening rate and learning attention of the first group of students were close to $100 \%$, and most students reported that all the 10 words were absorbed. In the second group, the learning attention and listening rate began to gradually decrease from the 15th word, and the students reported that on average, 13 words were absorbed. This experiment has proved the hypothesis 1, that is, the sense of acquisition of learning English words is proportional to the actual 
acquisition before reaching the critical point.

Hypothesis 2: Before reaching the critical point, the sense of acquisition is proportional to the actual gain. After reaching the critical point, if the sense of gain continues to increase, the sense of gain will decrease, which is inversely proportional to the actual gain, that is, the sense of acquisition is less than the actual gain. The hypothesis is a quadratic function with an opening down, the graph is " $\cap "$. Hypothesis proof through psychological experiment 2: The sense of acquisition of grabbing the red packets. Experimental design: This experiment adopts the cognitive psychology oral report method and is recorded by a scale. The instrument is a seven-point scale. with scores ranging from 1 to 7 indicating a range from very weak to very strong sense of acquisition. The survey tool is a questionnaire using a network survey software. The experimental date is May 4-5, 2017, and the experimental target group is the 2016 grade ethnic preparatory students from the school of ethnic education, Nanchang institute of science and technology. The sample size is 46 and the test content is 12 scale questions. The main question is "how you feel when you grabbed your first red packet online. In a continuous online red packets grabbing process, when you grab the first red envelope, what is your sense of acquisition? And what is your sense of acquisition when you grab the second, the third,...., the Nth?

Results analysis: The sense of acquisition of grabbing the red packet is zero when you grab no red packet. However, when you get your first hit the sense of gain is strongest at 6.72. When you grab the fifth red envelope, you get a saturation. At this point, the value reached a sub-high of 6.57.After that, with the increase of grabbing the red packets, the sense of gain gradually decreases. When it comes to the Nth red packet, the sense of gain decreases significantly, that is, the sense of gain shows a trend from low to high to low. Conclusion: Through this experiment, we have proved the hypothesis 2 .

Hypothesis 3: The sense of gain reaches the critical point at the very beginning where the sense of gain is the highest. After that, if the gain continues to increase, the sense of gain will decrease, which is inversely proportional to the actual gain, that is, the sense of gain is less than the actual gain. This hypothesis can be represented by an inverse scaling function and the graph is "।".Hypothesis proof through psychological experiment 3: The sense of acquisition of the national college entrance examination. The experimental design, method, measuring tool, object and experimental time are the same as above. The sample size is 71.The content of the test is 7 scale questions .The main question on the test is: "What is your sense of acquisition when you learned that you succeeded in the examination and were admitted to a university? What is the change of the sense of acquisition a month later? Two months later? Three months later? Six months later? Seven months later? The present moment?

Results analysis: The moment when you learned that you were admitted to the university, your sense of acquisition is the strongest. It peaked at the very beginning. After that, with the passage of time, there is a clear downward trend in the sense of acquisition, that is, from the beginning to the highest to the lowest. Conclusion: This experiment has proved hypothesis 3.

\section{The Investigation and Analysis of the Sense of Acquisition of the Ethnic College Students}

\subsection{Investigation on the sense of acquisition of the ethnic college students}

Questionnaire and scale design. The questionnaire consists of 57 questions, all of which are single choice questions. Among them, there are 50 scale scoring questions and 7 non-scale scoring questions. The questionnaire adopts a 7-point scale. 1 point for feeling "extremely weak", 2 points for feeling "very weak", 3 points for feeling "weak", 4 points for feeling "general", 5 points for feeling "strong", 6 points for feeling "very strong" and 7 points for feeling "extremely strong".

Data collection. A one-month online survey was conducted from March 12 to April 11, 2017 by using the network questionnaire survey tool. A total of 136 questionnaires were obtained, all of which were valid questionnaires. The respondents were mainly preppies in Nanchang institute of science and technology, accounting for $89.71 \%$. Freshmen account for $6.62 \%$, juniors $3.68 \%$, boys $33.09 \%$ and girls $66.91 \%$.There are also some formerly graduated students from the institute. 
Data analysis: The average score of 50 scale questions is from low to high, with a full score of 7.The highest score was 5.28 points for friendship, and the lowest score was 2.39 points for Gan dialect. The group difference was 2.89 points.

Among the top 10 senses of gain, the sense of friendship between friends and classmates of ethnic college students ranked first and second with 5.28 and 5.18 points respectively. The reason is that most of the students are the first time away from their parents to lead a life of independence, their biggest harvest in a year of preparatory study is having developed the ability to live independently. Their parents being not with them, they lack of an inner sense of security, so they try to obtain mind comfort and security from friends and classmates, and hopes to fill the vacancy caused by the absence of the parents. Therefore, they cherish this friendship very much. The third one on the list is computer literacy. It is now the information age. You can't get information without your cell phone and computer. And these cannot be separated from computer knowledge and software use. The main channel through which they acquire these skills is computer courses, so they have a strong sense of acquisition. Ranking 4-7 are health, study life, dormitory life and the sense of relationship between teachers and students. These factors are most closely related to students' study and life, so they have a higher sense of acquisition. Ranking 8-10 are No. 8 are sporting achievement, math and MHK certificate.

Among the last 10 senses of acquisition, the lowest is Gan dialect, with a score of 2.39.The reason is that students have little contact with it. Therefore, they have little understanding of it. Among the 10 senses of education acquisition, the highest score is computer course scored 5.01.Among the 9 senses of the second class acquisition, the highest is the national culture week scored 3.98.Among the 8 senses of gain of campus life, the sense of health is the highest scored 4.9.Among 11 senses of gain of Jiangxi culture, the red culture is the highest scored 4.19.

\subsection{Exploratory factor analysis of the sense of acquisition}

The initial factors were 55, and 12 factors were extracted after the dimension-reduction process of SPSS software, which jointly explained $74.129 \%$ and lost less information. KMO is 0.879 , indicating that it is suitable for factor analysis. The linear correlation of 12 factors is almost zero, which is consistent with the effect of factor analysis. Finally, through observation and analysis, 12 factors were further reduced to 6 factors: adaptive acquisition in Jiangxi, learning acquisition, skill acquisition, campus life acquisition, health acquisition and friendship acquisition.

\subsection{The analysis of verification factors of the sense of acquisition}

Reliability test of data. In this study, SPSS16.0 was used to study the internal consistency of data. Click the data to be analyzed in the left box one by one into the items box on the right. Using the Alpha model, click OK to get the results, and the Alpha coefficient is 0.960, indicating that the data used in this case has good reliability.

Validity test of data. Construct validity is a major measurement tool to measure validity, which reflects the degree of the internal structure of concepts and propositions. For the verification mechanism equation, if the theoretical model is well fitted with data, the structural validity is also good. For this study, it can be seen from the following group of fitting data that the theoretical model and data fit well, which proves that the structure validity is better.

The initial model: The model path originally designed took into account the main logical relationships of six potential variables, but it ignored the impact of friendship on health and school life on learning and skills. In real life, friendship does have an effect on health. Campus life includes community life, second class life, library life, etc. These activities will have certain influence on learning and skills. Therefore, the model is improved and revised several times until it meets the minimum fitting index: chi-squared dropped from 403 to 377; GFI rose from 0.765 to 0.792, indicating that the change was correct and successful. However, compared with the qualified indicators, the fitting indexes are still not ideal. The chi-square value is still relatively large, RMSEA is greater than 0.05, and GFI, NFI, TLI and CFI values all fail to meet the theoretical requirements of above 0.9 .So the model is revised again. The principle of correction is: first, the adjustment on the path correlation of potential variables will improve the fitting degree of the model, 
and then the model will be adjusted from the path association of measurable variables to improve the fitting degree. Because the path fit of potential variables is the basis of the whole model, it determines the direction and the pros and cons of the model, which is of more theoretical and practical significance. Finally, the final model is determined after repeated modifications .All the fitting indexes except RMR have reached and exceeded the qualified standard, which indicates that the structural equation fitting degree is good. The successful fitting of structural equation verifies the correctness of model hypothesis and the correlation of factors.

\section{Conclusion}

Based on the theories of cognitive science, information processing, pedagogy, etc and with the approaches of empirical research and exploratory factors and confirmatory factors analysis, this essay has made a comprehensive study and analysis on the factors influencing the ethnic college students in Jiangxi province. It raised three hypotheses and has proved them by making a series of psychological experiments. Through SPSS exploratory factor analysis, 6 representative factors are found out from 57 acquired sensitivity factors. Then, through Amos confirmatory factor analysis, the constructed model is fitted. Through modification, the requirements of fitting are reached, and the six representative factors are finally proved: Namely, the adaptive acquisition in Jiangxi, the learning acquisition, the skills acquisition, the campus life acquisition, the health acquisition and the friendship acquisition.

\section{References}

[1] Liu jiqing. Education reform based on "sense of acquisition" [J]. Development research of education, July 2017

[2] Wang shushi. A brief discussion on the sense of acquisition [J]. Daqing social science, August 2016

[3] Zhang pin. Theoretical connotation and contemporary value of "sense of acquisition" [J]. Journal of Henan university of technology (social science edition), December 2016

[4] Wang jibing.School education fulfills one's sense of acquisition [J]. Management of primary and secondary schools, July 2015 